\title{
Topological surface states scattering in antimony
}

\author{
Awadhesh Narayan, Ivan Rungger, and Stefano Sanvito \\ School of Physics and CRANN, Trinity College, Dublin 2, Ireland \\ (Received 19 July 2012; revised manuscript received 21 September 2012; published 5 November 2012)
}

\begin{abstract}
In this work we study the topologically protected states of the $\mathrm{Sb}(111)$ surface by using ab initio transport theory. In the presence of a strong surface perturbation we obtain standing-wave states resulting from the superposition of spin-polarized surface states. By Fourier analysis, we identify the underlying two dimensional scattering processes and the spin texture. We find evidence of resonant transmission across surface barriers at quantum well state energies and evaluate their lifetimes. Our results are in excellent agreement with experimental findings. We also show that despite the presence of a step edge along a different high-symmetry direction, the surface states exhibit unperturbed transmission around the Fermi energy for states with near to normal incidence.
\end{abstract}

DOI: 10.1103/PhysRevB.86.201402

PACS number(s): 73.63.-b, 73.20.-r, 73.50.Bk, 73.22.-f

Introduction. In the past few years topological insulators (TIs) have become an intensely studied field of condensed matter physics. ${ }^{1,2}$ The unique metallic states at the surface of a TI can be used both as a tabletop playground to prove fundamental concepts developed for particle physics and also as a new materials platform for intriguing quantum applications in both spin electronics and quantum computing. ${ }^{3-5}$ The first three-dimensional TI to be experimentally realized was a $\mathrm{Bi}-\mathrm{Sb}$ alloy, ${ }^{6}$ following a theoretical prediction by $\mathrm{Fu}$ and Kane. ${ }^{7}$ The topological nature of the alloy is inherited from the parent element antimony, which has a nontrivial principal topological invariant $v_{0}$, whereas bismuth has a trivial $Z_{2}$ invariant. $^{8}$

Although Sb itself is a semimetal, its (111) surface hosts two spin-polarized bands, which extend around the Fermi energy $E_{\mathrm{F}}$. These form a single distorted Dirac cone where the lower bands are lifted upwards. The electronic properties of $\mathrm{Sb}(111)$ thin films have been investigated both theoretically and experimentally. ${ }^{9-12}$ In a recent experiment, Seo et al. demonstrated that the topological surface states are extraordinarily insensitive to the presence of surface barriers. ${ }^{13}$ They probed the extended nature of $\mathrm{Sb}(111)$ surface states by using a scanning tunneling microscope (STM) and found that these transmit across surface atomic steps with a high probability. Their analysis of the standing-wave states on surface terraces revealed the novel chiral spin texture of the two surface states, consistent with earlier angle-resolved photoemission spectroscopy (ARPES) measurements. ${ }^{14}$

In this Rapid Communication we seek to theoretically recreate the above-mentioned experiment by using $a b$ initio transport theory, and show that we can reproduce the formation of quantum well states and their lifetimes, as well as the wavelengths and phase shifts of the scattering states. Thereby we demonstrate that by first-principles calculations one can describe the correct scattering properties of such topologically protected surface states. In addition to comparing our results favorably to the experiments, we predict the scattering properties of these states in the presence of a surface perturbation along a direction orthogonal to the one probed experimentally.

Computational methods. Our first-principles electronic structure calculations are performed with density functional theory (DFT) using the local density approximation (LDA) to the exchange-correlation functional. We employed the SIESTA package, which implements a linear combination of atomic orbital basis set. ${ }^{15}$ Spin-orbit interaction, essential to describe the surface states, has been included via the on-site approximation. ${ }^{16}$ We include $\mathrm{Sb} 5 s$ and $5 p$ as the valence electrons. In the slab geometry, there is a $10 \AA$ vacuum in the supercell to avoid interaction between periodic images. The transport properties are then calculated by using SMEAGOL, which combines the nonequilibrium Green's function (NEGF) method with DFT. ${ }^{17-19}$ In SMEAGOL the scattering region is attached to one or more semi-infinite electrodes via self-energies. The charge density is calculated by integrating the nonequilibrium Green's function, along a contour in the complex energy plane. For this we use 16 energy points on the complex semicircle, 16 points along the line parallel to the real axis, and 16 poles. Periodic boundary conditions are employed in directions orthogonal to the transport direction, while using open boundary conditions along the transport direction allows us to simulate single scatterers. ${ }^{20}$ We use an equivalent temperature of $300 \mathrm{~K}$ for broadening in the Fermi distribution. Our order- $N$ implementation of the code allows us to treat large systems. ${ }^{20}$ We use a double- $\zeta$ polarized (DZP) basis set, with a cutoff energy of 300 Ry for the real space mesh. We have carefully checked the convergence of our results with respect to all the parameters used.

$\mathrm{Sb}$ crystallizes in a rhombohedral structure [space group $D_{3 d}^{5}(R \overline{3} m)$ ] with two atoms per unit cell. An alternate way to represent its structure is in an hexagonal setting with the unit cell comprising six atoms. This representation is particularly useful to construct two-dimensional slabs, which are made of Sb bilayers, as shown in Fig. 1(a). The interbilayer coupling is weak and it is possible to create surface steps which are a single bilayer high. ${ }^{13,21}$ The bulk structure was relaxed using the Vienna $a b$ initio simulation package (VASP), ${ }^{22}$ until the forces were less than $0.01 \mathrm{eV} / \AA$.

Results. We begin by calculating the surface band structure of 6- and 12-bilayer-thick slabs of Sb [Figs. 1(b) and 1(c)], by using a $10 \times 10$ in-plane $k$-point grid. The distorted Dirac cone at $\bar{\Gamma}$ is gapless, indicating minimal interaction between the top and bottom surfaces of the slab. The surface band structure matches well previous $a b$ initio calculations. ${ }^{9,10,12}$ We find the Dirac point at an energy of about $210 \mathrm{meV}$ below $E_{\mathrm{F}}$ for 6- and 12-bilayer slabs. In order to simulate the ARPES spectrum of an infinitely thick slab we perform a SMEAGOL calculation for the 6-bilayer slab, where we attach semi-infinite Sb electrodes at the bottom layer via self-energies. The ARPES spectrum is then obtained by calculating the projected density of states on 


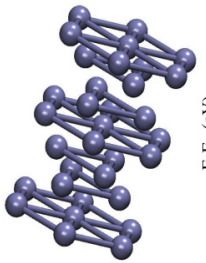

(a)

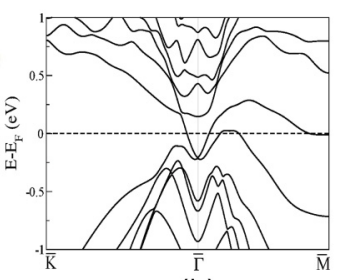

(b)

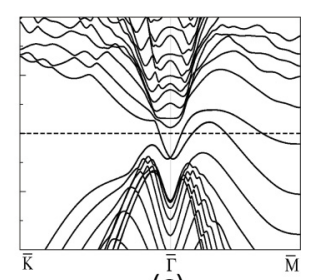

(c)

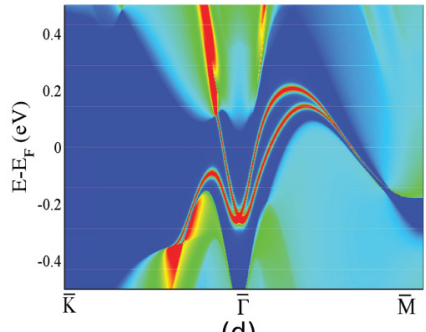

(d)

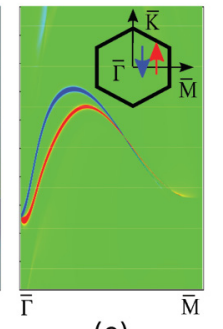

(e)

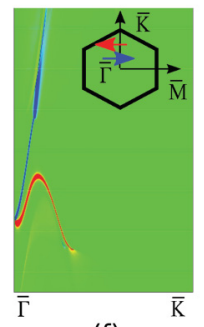

(f)
FIG. 1. (Color online) (a) Structure of antimony in the hexagonal setting. The atoms form a bilayer structure with the intralayer distance as $1.51 \AA$ and the interlayer distance as $2.25 \AA$. Band structure for (b) 6- and (c) 12-bilayer-thick slabs along $\bar{K}-\bar{\Gamma}-\bar{M}$ directions. (d) Simulated ARPES from a semi-infinite slab. The distorted Dirac cone is found to comprise spin-polarized surface bands. Here and henceforth warmer colors represent higher PDOS (red represents largest values, blue lowest ones, with the color scale in between being linear). Spin-resolved ARPES along (e) $\bar{\Gamma}-\bar{M}$ and (f) $\bar{\Gamma}-\bar{K}$ directions showing the opposite spins of the two surface bands along the directions indicated by arrows in the inset of the figures. In this case, red and blue colors indicate up and down spins, respectively.

the surface atoms, and the result is shown in Fig. 1(d). The spin-resolved ARPES [Fig. 1(e) and 1(f)] shows that the two surface bands carry opposite spin and exhibit the characteristic spin texture associated with topologically nontrivial materials. Furthermore, it can be seen that the surface states are more pronounced close to the $\bar{\Gamma}$ point. Once the two bands turn downwards from their maximum point, they are less localized on the surface due to their hybridization with the bulk bands. This matches with ARPES experiments, where at $\bar{M}$, no surface states are found. ${ }^{14}$ Thus, in both scattering and ARPES experiments, one would expect the dominant contributions to come from an area around the center of the Brillouin zone (BZ), with a radius of about one third of the length of BZ along the $\bar{\Gamma}-\bar{M}$ direction. We note that the good agreement with the ARPES experiments shows that the LDA exchange-correlation functional is appropriate for this system.

Next we simulate a step perturbation on the surface for two different directions: In the first case the step runs along the $y$ direction [see Fig. 2(b)], so that the transport is along the $x$ direction, which is the same as in the experiment in Ref. 13; and as a second orientation we choose the orthogonal direction (step running along $x$ ) to evaluate the effect of the orientation on the scattering. We have relaxed the step geometry for a smaller scattering region, but the atomic displacements were only minor, and henceforth we use the unrelaxed step configuration. A single-bilayer-high step is created over a length of $120 \AA$. The adjacent flat region extends over $270 \AA$. The setup consists of a 13-bilayer-thick region with a short 12-bilayer-thick region on the left and a longer
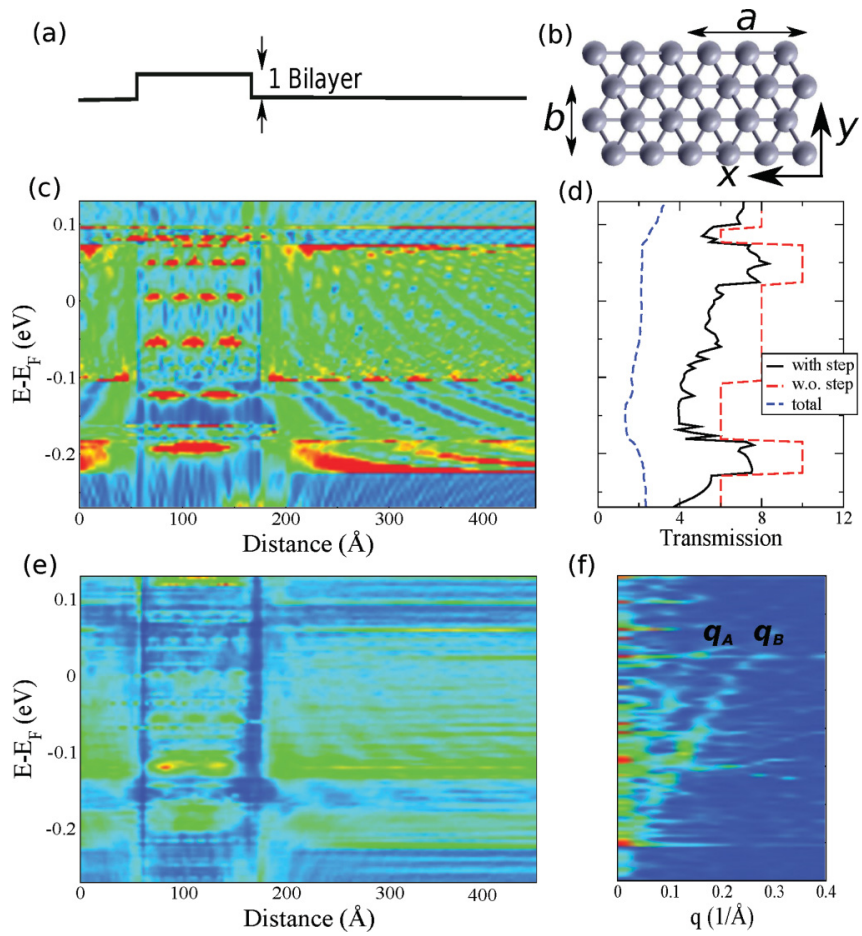

FIG. 2. (Color online) (a) A schematic of the setup showing onebilayer-high surface perturbation on a 12-bilayer slab. (b) Top view of the slab; surface step runs along $y$ for the first setup and along $x$ for the second. (c) PDOS for surface atoms with a single step adjacent to a flat region for the first setup at $k_{\perp}=0$. The quantization of the energy levels in the step is clearly seen, with accompanying phase shifts in the adjacent flat region. (d) Transmission at $k_{\perp}=0$ with and without the step, indicating finite scattering due to the step. Average of transmission over all $k_{\perp}$ points is also shown. (e) PDOS for surface atoms averaged over all $k_{\perp}$. (f) Fourier transform of PDOS data in (e) over the flat region reveals the different allowed scattering wave vectors. The most prominent features, $q_{A}$ and $q_{B}$, are nearly linear with slopes equal to $1.1 \mathrm{eV} \AA$.

one on the right, as shown schematically in Fig. 2(a) [for the actual setup see the Supplemental Material $\left.(\mathrm{SM})^{23}\right]$. We attach semi-infinite leads on the left- and right-hand sides of the scattering region, by means of the self-energies calculated by SMEAGOL. This enables us to simulate isolated scatterers, thus avoiding the use of periodic boundary conditions along the transport direction. ${ }^{20}$

The total projected density of states (PDOS) $\mathcal{N}_{\text {total }}$ is obtained by integrating over all $k_{\perp}$ points perpendicular to the transport direction

$$
\mathcal{N}_{\text {total }}(E)=\int_{k_{\perp}} \mathcal{N}_{k_{\perp}}(E) d k_{\perp} .
$$

Analogously the total transmission is given by $T_{\text {total }}(E)=$ $\int_{k_{\perp}} T_{k_{\perp}}(E) d k_{\perp}$. We note that in the first orientation of the step $k_{\perp}$ runs along the $\bar{\Gamma}-\bar{K}$ direction and the transport direction is parallel to $\bar{\Gamma}-\bar{M}$ in reciprocal space, whereas in the second orientation $k_{\perp}$ runs along $\bar{\Gamma}-\bar{M}$ and the transport direction is parallel to $\bar{\Gamma}-\bar{K}$. While we find that three $k_{\perp}$ points are sufficient for obtaining a converged self-consistent potential, we need many more $k_{\perp}$ points for accurately integrating $\mathcal{N}_{\text {total }}$ for a given potential, where we therefore use $200 k_{\perp}$ points. 
The $\mathcal{N}_{k_{\perp}}$ of the atoms on the top surface for $k_{\perp}=0$ is shown in Fig. 2(c). The quantum well states formed by quantization of the energy levels in the step region are clearly visible, and extend over the energy window in which the two topological surface bands exist. The PDOS on the adjacent flat region shows oscillatory behavior typical of one-dimensional scattering barriers, ${ }^{20}$ and it has phase shifts at energies corresponding to the allowed energy states in the step region. At those energies we also find resonant transmission across quantum well states, visible as peaks in the transmission curve in Fig. 2(d). This matches the experimental observation of resonant tunneling through the surface barrier at those energies. It indicates a remarkably long phase coherence length of hundreds of angstroms, which is due to the extended nature of topological surface states. ${ }^{13}$ Further, the change of phase when the states are reflected from the barrier is nearly zero. Over the entire energy range there is clearly a rather large amount of scattering caused by the step and the transmission drops significantly below the value in the absence of step.

We have verified that the single bilayer step at the top surface perturbs the bottom layer minimally and there is only small coupling between the two surfaces even in the presence of the surface step. ${ }^{23}$

The integrated $\mathcal{N}_{\text {total }}$ is shown in Fig. 2(e). The main features corresponding to the quantized energy levels can still be identified at almost the same energies found for $k_{\perp}=0$, but are broadened and less pronounced. The broadening of energy levels for $k_{\perp}=0$ is $8-12 \mathrm{meV}$, while for the total it increases by a factor of about 3 . In experiment, these were found to lie between 20 and $45 \mathrm{meV}^{13}$ Hence, the states lifetimes, which are inversely proportional to their broadening, agree quantitatively with those found experimentally. This shows that the scattering properties of the step are well reproduced in our calculations. We note that quantitatively the results for a 12-bilayer slab differ somewhat from the ones one would obtain for a semi-infinite surface due to the different surface band structures [Fig. 1(c) versus 1(d)]. First, the linear dispersion region of the surface states around $\Gamma$ is more extended in energy for the semi-infinite surface, and this results in quantum well state spacing remaining constant, whereas for the 12-bilayer slab the spacing reduces with increasing energy. Moreover, for 12 bilayers we find band edges at -185 and $-108 \mathrm{meV}$, which are absent in the semi-infinite system, and which give strong contributions to the 12-bilayer PDOS. Due to the enhanced PDOS at these band edges the phase shifts corresponding to resonant tunneling are not as clearly pronounced for $\mathcal{N}_{\text {total }}$ as for $k_{\perp}=0$. Finally, whereas for the semi-infinite slab there are only two discrete surface bands around $E_{F}$, and all the other bands are diffuse, for the 12-bilayer slab clearly the number of distinct bands is larger, which, as we will show, leads to many more features in the scattering. These lead to discrete short-wavelength scattering processes which would be absent for a semi-infinite slab.

In order to analyze the scattering mechanism and probe the chiral spin texture of the surface bands, we evaluate the Fourier transform (FT) of $\mathcal{N}_{\text {total }}$ along the flat region on the right-hand side of the step, and the result is shown in Fig. 2(f). For a given energy and $k_{\perp}$ ( $k_{\perp}$ is conserved during scattering) there are standing waves with a wave vector $q$ for every pair of scattering states in the $\mathrm{Sb}$ electrodes with opposite group velocities. For each such pair of states with indices $i$ and $j$ we get the norm of scattering vector $q_{i j}=\left|k_{i}-k_{j}\right|$, at which therefore the FT has enhanced amplitude. In Fig. 2(f) we find a rather large amount of noise at low $q$, partly due to the fact that we integrate $\mathcal{N}_{\text {total }}$ over a finite number of $k_{\perp}$ points, nevertheless we can identify two prominent features that are preserved for increasing $q$. The first is an enhanced amplitude starting at about $-210 \mathrm{meV}$ and then increasing linearly with $q$ with a slope of $1.1 \mathrm{eV} \AA$, and the second is an equivalent enhancement with the similar slope starting at $-110 \mathrm{meV}$. These two prominent scattering wave vectors are also found in the experiments of Seo et al., where they are labeled $q_{B}$ and $q_{A}$, respectively. $q_{B}$ is attributed to scattering between the surface states close to $\bar{\Gamma}$ having the same spin, but opposite momentum direction. In this case the scattering state momenta are not equal, unlike in conventional scattering, because of the unique spin texture of the surface states resulting in an asymmetric band structure for a given spin. The other scattering wave vector $\left(q_{A}\right)$ is attributed to scattering between neighboring hole pockets away from $\bar{\Gamma}$. The calculated slopes of $q_{A}$ and $q_{B}$ are remarkably close to the value of $1.2 \mathrm{eV} \AA$ found experimentally. ${ }^{13}$ We note that to explain the origin of both $q_{A}$ and $q_{B}$, one needs to go beyond the one-dimensional model invoked by Seo et al. and a two-dimensional treatment is required.

An STM experiment measures the total scattering in all the reciprocal space directions. In order to establish which $k_{\perp}$ points give rise to the $q_{A}$ and $q_{B}$ features in the average, we decompose the allowed scattering processes along different $k_{\perp}$ directions. To illustrate the general scattering mechanism, in Fig. 3(c) we show the Fourier transform for an arbitrary $k_{\perp}$ point, and the bands of the $\mathrm{Sb}$ electrodes along the transport direction for the same $k_{\perp}$. As an example, we consider the energy at $-150 \mathrm{meV}$, where in the band structure we find two bands crossing for positive $k$, at $k_{1}$ and $k_{2}$. In the Fourier transform we find three scattering vectors: $q_{1}=k_{2}-k_{1}$, $q_{2}=2 k_{1}$ (obtained by $k_{1}$ scattering to $-k_{1}$ ), and similarly $q_{3}=2 k_{2}$. The amplitude is highest towards the band edge, where the PDOS is maximal. Analyzing the FT for all $k_{\perp}$ points, we find that most such features found for a single $k_{\perp}$ disappear when averaging, except for $q_{A}$ and $q_{B}$. This shows that the scattering processes visible in STM experiments are only a small subset of all processes occurring. Therefore no visible scattering from defects in an STM experiment does not imply perfect transmission, since the absence of standing-wave patterns can also be due to the fact that the features may be broadened upon integration over $k_{\perp}$, even for substantial scattering for each single $k_{\perp}$.

There are two key factors which decide which $q$ vectors dominate. First, for a given $k_{\perp}$ there needs to be a high scattering between the initial $\left(k_{i}\right)$ and final $\left(k_{j}\right)$ states. This is the case if the spins in the two states are aligned and if the surface PDOS for them is large. This, therefore, excludes bulk states and states with opposite spins. Second, for the features to remain prominent when integrated over $k_{\perp}$, it is necessary that there is an extended region in the $\mathrm{BZ}$ where these features are found more or less unchanged. This is true when the change of band structure along $k_{\perp}$ is small, which is the case close to a maximum or a minimum, i.e., when $\partial E / \partial k_{\perp}=0$, and ideally when the band curvature is small $\left(\partial^{2} E / \partial k_{\perp}^{2}\right.$ is small). We have verified that the above two conditions are 
(a)

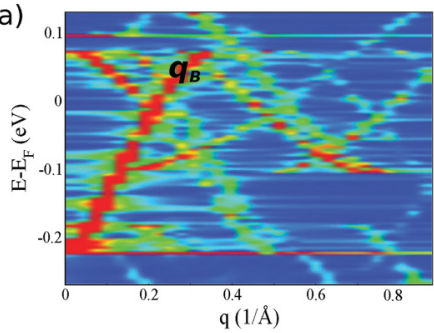

(b)

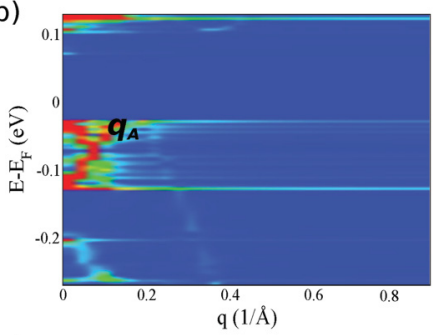

(c)

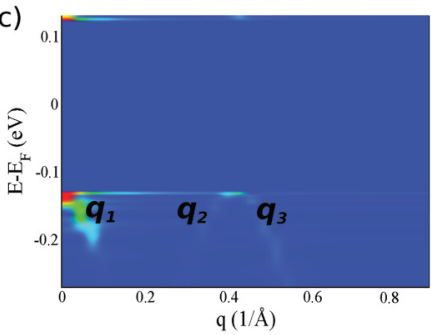

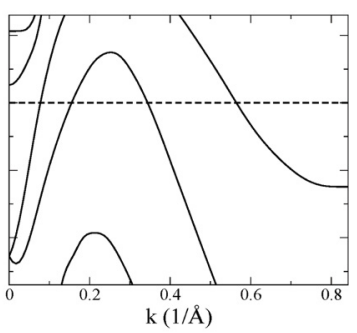
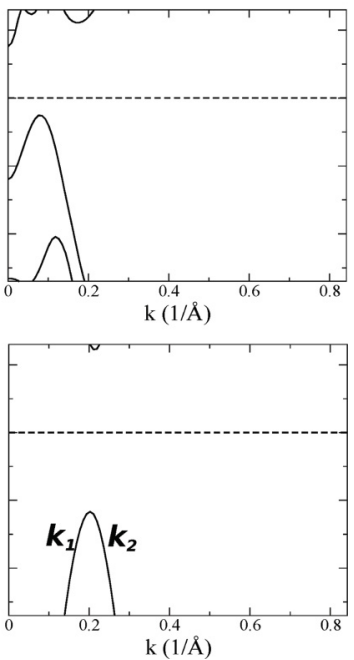

FIG. 3. (Color online) The allowed scattering wave vectors obtained from the Fourier transform of PDOS data at (a) $k_{\perp} b=0$, (b) $k_{\perp} b=0.16 \pi$, and (c) $k_{\perp} b=0.50 \pi$. The panels on the right show the corresponding band structures at the same $k_{\perp}$ for the 12-bilayer slab electrodes.

indeed satisfied for $q_{A}$ and $q_{B}$. From the 12-layer slab band structure along $\bar{\Gamma}-\bar{K}$ [Fig. 1(c)] we identify two such features: The first is the minimum at about $k_{\perp}=\bar{\Gamma}$, and the second is the maximum at $k_{\perp} b=0.16 \pi$. In Figs. 3(a) and 3(b) we show the scattering wave vectors for these two $k_{\perp}$. We clearly identify the features leading to $q_{A}$ and $q_{B}$. The remaining features in the FT disappear under averaging, since for these the aforementioned conditions are not satisfied. We note that a related study has been recently performed by Takane and Imura by using a low-energy Dirac theory, where they find perfect transmission at all incidence angles for a hyperbolic step. ${ }^{24}$ However, as noted by the authors, their analysis is valid in the long wavelength regime, while we focus on atomic-scale terraces. Our results clearly demonstrate that there is scattering between states on the same Dirac cone for $k_{\perp} \neq 0$, which leads to the appearance of the $q_{A}$ scattering vector. The fact that such a scattering vector is found prominently also in experiments indicates that for non-normal incidence the states are not perfectly transmitted, in agreement with our findings.

Finally, we create a step on the $\mathrm{Sb}(111)$ surface along the $x$ direction. In this case the slab consists of six bilayers, since simulating a 12-bilayer scattering region along this direction is beyond our computational resources (see SM for a description of the setup ${ }^{23}$ ). This direction is promising since there is an energy window [ -60 to $20 \mathrm{meV}$ in Fig. 1(b)] over which only a single spin-polarized surface state exists for $k_{\perp}=0$ (note that in this case the transport direction is parallel to the $\bar{\Gamma}-\bar{K}$ line in reciprocal space), which is reminiscent of

(a)

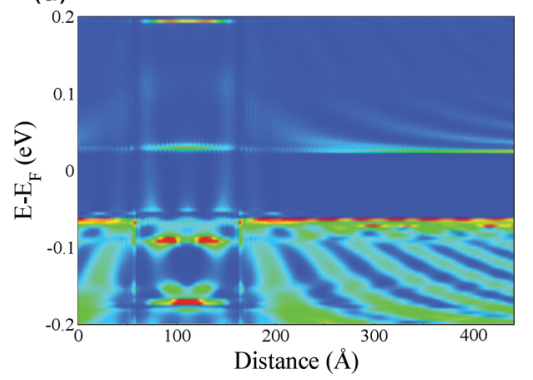

(b)

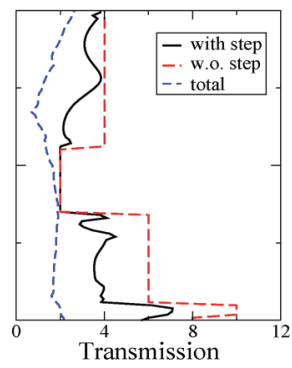

FIG. 4. (Color online) (a) PDOS at $k_{\perp}=0$ for surface atoms with a single step adjacent to a flat region, with the step extending along the $x$ direction in Fig. 2(b). There is an energy region from -60 to $20 \mathrm{meV}$ with no scattering and hence no standing-wave states. (b) Transmission at $k_{\perp}=0$, indicating the perfect transmission around $E_{F}$ even in the presence of a surface barrier, the defining feature of a topological state. Total transmission also shows minimal scattering in that energy range.

the prototypical TI $\mathrm{Bi}_{2} \mathrm{Se}_{3}$. The $\mathcal{N}_{k_{\perp}}$ of the surface atoms for $k_{\perp}=0$ is plotted in Fig. 4(a). In the energy range of a single spin-polarized state there is no scattering, which is the hallmark of a topological surface state. From -170 to $70 \mathrm{meV}$ quantum well states are found as a result of superposition between the two surface bands, with a mechanism analogous to that in the first step orientation. Figure 4(b) shows the transmission at $k_{\perp}=0$ with and without the surface step. Remarkably, there is a perfect transmission in this energy window, despite the presence of the strong surface perturbation in the form of an extended single-bilayer-high surface step. This can be explained by invoking the general principle that disorder which does not break time reversal symmetry cannot localize a single topologically protected surface state. Away from this energy region, there is substantial scattering caused by the step and the transmission drops down from the value in its absence. The total transmission averaged over all $k_{\perp}$ points is reduced for all energies, and one can expect that as the amount of disorder increases, the total transmission will be dominated by small $k_{\perp}$ contributions. We believe that these findings would provide a strong motivation for study of $\mathrm{Sb}$ surface with steps along the $\bar{\Gamma}-\bar{K}$ direction.

Summary. In conclusion, we have performed an ab initio study of the topological surface states on the $\mathrm{Sb}(111)$ surface and their response to the presence of single-bilayer-high steps, showing excellent agreement with experimental observations. We have identified the various scattering processes possible and formulated general conditions that lead to the formation of the dominant scattering features. This enabled us to confirm the fascinating chiral spin texture of the surface states. Resonant tunneling transmission across surface barriers, indicative of the extended nature of these states, was found. We identified phase shifts in the scattered PDOS at quantum well state energies and evaluated their lifetimes. The results demonstrate that it is possible to fully characterize the scattering properties of the barriers with first-principles calculations. Finally, we have shown that one can have minimal scattering along other high-symmetry directions even in presence of strong surface perturbations, which provides a unique signature for 
the topologically protected nature of these states. We believe that this can be readily tested in future experiments.

Acknowledgments. This work is financially supported by the Irish Research Council for Science, Engineering and Technology (IRCSET) under the EMBARK initiative. Computational resources have been provided by the Trinity Centre for High Performance Computing (TCHPC). I.R. and S.S. acknowledge additional financial support by KAUST (ACRAB project). The authors would like to thank Anna Pertsova for illuminating discussions.
${ }^{1}$ M. Z. Hasan and C. L. Kane, Rev. Mod. Phys. 82, 3045 (2010).

${ }^{2}$ X.-L. Qi and S.-C. Zhang, Rev. Mod. Phys. 83, 1057 (2011).

${ }^{3}$ L. Fu and C. L. Kane, Phys. Rev. Lett. 100, 096407 (2008).

${ }^{4}$ X.-L. Qi, R. Li, J. Zang, and S.-C. Zhang, Science 323, 1184 (2009).

${ }^{5}$ D. Pesin and A. H. MacDonald, Nat. Mater. 11, 409 (2012).

${ }^{6}$ D. Hsieh, D. Qian, L. Wray, Y. Xia, Y. S. Hor, R. J. Cava, and M. Z. Hasan, Nature (London) 452, 970 (2008).

${ }^{7}$ L. Fu and C. L. Kane, Phys. Rev. B 76, 045302 (2007).

${ }^{8}$ J. C. Y. Teo, L. Fu, and C. L. Kane, Phys. Rev. B 78, 045426 (2008).

${ }^{9}$ S.-X. Wang, P. Zhang, and S.-S. Li, arXiv:1201.1976.

${ }^{10}$ P.-F. Zhang, W. Duan, J. Wu, and Z. Liu, Phys. Rev. B 85, 201410(R) (2012).

${ }^{11}$ K. Sugawara, T. Sato, S. Souma, T. Takahashi, M. Arai, and T. Sasaki, Phys. Rev. Lett. 96, 046411 (2006).

${ }^{12}$ G. Bian, X. Wang, Y. Liu, T. Miller, and T.-C. Chiang, Phys. Rev. Lett. 108, 176401 (2012).

${ }^{13}$ J. Seo, P. Roushan, H. Beidenkopf, Y. S. Hor, R. J. Cava, and A. Yazdani, Nature (London) 466, 343 (2010).

${ }^{14}$ D. Hsieh, Y. Xia, L. Wray, D. Qian, A. Pal, J. H. Dil, J. Osterwalder, F. Meier, G. Bihlmayer, C. L. Kane, Y. S. Hor, R. J. Cava, and M. Z. Hasan, Science 323, 919 (2009).
${ }^{15}$ J. M. Soler, E. Artacho, J. D. Gale, A. Garcia, J. Junquera, P. Ordejón, and D. Sánchez-Portal, J. Phys.: Condens. Matter 14, 2745 (2002).

${ }^{16}$ L. Fernández-Seivane, M. A. Oliveira, S. Sanvito, and J. Ferrer, J. Phys.: Condens. Matter 18, 7999 (2006).

${ }^{17}$ A. R. Rocha, V. M. Garcia-Suarez, S. Bailey, C. Lambert, J. Ferrer, and S. Sanvito, Nat. Mater. 4, 335 (2005).

${ }^{18}$ A. R. Rocha, V. M. Garcia-Suarez, S. Bailey, C. Lambert, J. Ferrer, and S. Sanvito, Phys. Rev. B 73, 085414 (2006).

${ }^{19}$ I. Rungger and S. Sanvito, Phys. Rev. B 78, 035407 (2008).

${ }^{20}$ B. Naydenov, M. Mantega, I. Rungger, S. Sanvito, and J. J. Boland, Phys. Rev. B 84, 195321 (2011).

${ }^{21}$ K. K. Gomes, W. Ko, W. Mar, Y. Chen, Z.-X. Shen, and H. C. Manoharan, arXiv:0909.0921.

${ }^{22}$ G. Kresse and J. Furthmuller, Phys. Rev. B 54, 11169 (1996).

${ }^{23}$ See Supplemental Material at http://link.aps.org/supplemental/ 10.1103/PhysRevB.86.201402 for the scattering setup and PDOS on the bottom layer of the slabs.

${ }^{24}$ Y. Takane and K.-I. Imura, J. Phys. Soc. Jpn. 81, 093705 (2012). 